\title{
MÉTODO MANUAL PARA DEPOSIÇÃO DE TINTAS FOTOLUMINESCENTES BASEADAS EM LOFS MISTAS APLICADO A CODIFICAÇÃO DE DOCUMENTOS
}

\author{
Millayd M. Nogueira ${ }^{a}$, Luiz H. M. Costa ${ }^{a}$, Jakson N. C. Reis ${ }^{a}$, Antonio Marcos U. Araújo ${ }^{\mathrm{b}}$, Érico de Moura Neto ${ }^{\mathrm{a}, * \text {, }}$ e \\ Jarley F. S. Nascimento ${ }^{a}$ \\ anstituto Federal de Educação, Ciência e Tecnologia do Rio Grande do Norte, 59500-000 Macau - RN, Brasil \\ bUniversidade Federal do Rio Grande do Norte, 59078-970 Natal - RN, Brasil
}

Recebido em 06/04/2020; aceito em 30/07/2020; publicado na web em 10/09/2020

\begin{abstract}
MANUAL METHOD FOR THE DEPOSITION OF PHOTOLUMINESCENT INKS BASED ON MIXED LOFs APPLIED TO DOCUMENT ENCONDING. In this work, the process of synthesis of Metal-Organic Frameworks containing lanthanide ions (LOFs), accelerated via a precipitating agent, capable of accelerating the process of nucleation of MOF crystals, in order to explore the optical properties of this precursor solution (SP_Gd/EU-MOF), as a quick-response photoluminescent material in the coding of official documents. The SP_Gd/Eu-MOF was deposited on the paper substrates manually, using pilot board master liquid ink pens with medium tip. The SP_Gd/Eu-MOF, was characterized by photoluminescence spectroscopy after crystallization on a vial and at surface of the papers, and the spectra showed identical spectral profiles, showing that the interaction with the substrate surface did not promote significant changes in the site symmetry of $\mathrm{Eu}^{3+}$ ions. After being deposited, the material was excited by UV radiation $(\lambda=254 \mathrm{~nm})$, and emitted the red color characteristic of the $\mathrm{Eu}^{3+}$ ion. The SP_Gd/Eu-MOF, showed good mechanical stability in the abrasiveness test carried out with a rubber remaining deposited on the substrate, demonstrating it is potential to act as proof of authenticity in the encoding of documents, revealing itself as another low cost alternative for the deposition of photoluminescent inks.
\end{abstract}

Keywords: MOFs; LOFs; inks; gadolinium; europium.

\section{INTRODUÇÃO}

A síntese de materiais inovadores com propriedades luminescentes está promovendo o desenvolvimento de novos dispositivos ópticos. ${ }^{1-3}$ Nesse quesito, as Redes Metalorgânicas (MOFs - Metal-Organic Framework), surgem como uma alternativa. As MOFs contendo íons lantanídeos (LOFs - Lanthanide-Organic Framework), vêm recebendo nos últimos anos grande atenção devido as propriedade únicas dos íons lantanídeos, combinada a grande diversidade estrutural apresentada por estes materiais. ${ }^{4-11}$

A segurança tornou-se indispensável, especialmente no que diz respeito aos documentos oficiais, pois a falsificação de documentos viola as leis de direitos autorais e causa enormes prejuízos. ${ }^{2,3}$ Grupos de pesquisa reportaram na literatura, a utilização de MOFs contendo íons lantanídeos em processos de anti-falsificação via impressoras a jato de tinta., ${ }^{3,-14}$

Códigos de segurança são características incorporadas a documentos e também ao papel moeda, com a finalidade de garantir maior autenticidade quanto a sua integridade e sua origem, onde alguns fatores determinam quais e quantos elementos devem ser inseridos como por exemplo, o valor do documento, a função e frequência com que será utilizado. ${ }^{15}$ A extensão da variedade de componentes utilizados abrange desenhos, textos, tintas, holografias e dispositivos ópticos variáveis. ${ }^{15}$

Jiang et al. ${ }^{16}$ relataram pela primeira vez um material que se baseia em um conjunto de pontos de carbono luminescentes (CD) preparados a partir de m-fenilenodiamina e álcool polivinílico (PVA), com emissões de modo triplo simultaneamente. A dispersão aquosa, após ser inserida nas canetas esferográficas gel e depositada por nos substratos, não era completamente invisível a luz do dia. No entanto, demonstrou elevado potencial para atuar como tinta anti-falsificação avançada permitindo autenticação óptica por três vias diferentes,

*e-mail: erico.neto@ifrn.edu.br fotoluminescência (PL), conversão ascendente de energia (UCPL) e fosforescência a temperatura ambiente (RTP).

Foi proposta por Wang e colaboradores ${ }^{17}$ uma nova estratégia de codificação com MOFs em nanoescala (NMOFs) como unidade de armazenamento de sinal com múltiplas cores contra falsificações. Foram relatados MOFs emissores de luz vermelha e verde, sintetizados com ácido 5-boronoisoftálico (5-bop) e ácido isoftálico (1,3-BDC) como ligantes mistos e os íons luminescentes európio $\left(\mathrm{Eu}^{3+}\right)$ e terbio $\left(\mathrm{Tb}^{3+}\right)$, que foram depositados via impressoras a jato de tinta e através de canetas rollerball M\&G com diâmetros de $0,5 \mathrm{~mm}$.

Neste trabalho, reportaremos um método eficaz de síntese e aplicação de soluções precursoras de LOFs mistas contendo os íons $\mathrm{Gd}^{3+}$ e $\mathrm{Eu}^{3+}$ (SP_Gd/Eu-MOF), para atuarem como tintas fotoluminescentes através da deposição de canetas de tinta líquida pilot board master com ponta média de forma manual, método ainda não relatado na literatura. A SP_Gd/Eu-MOF, após sua secagem nas folhas, ficou completamente invisível à luz do dia e mostrou ser eficaz para atuar na codificação de documentos, emitindo a coloração vermelha que é característica das transições ${ }^{5} \mathrm{D}_{0} \rightarrow{ }^{7} \mathrm{~F}_{\mathrm{j}}$ dos íons $\mathrm{Eu}^{3+}$, revelando-se uma alternativa eficiente e de baixissimo custo, para construir um sistema de codificação para garantir a autenticidade de documentos oficiais, o que se constitui ainda nos dias atuais como um desafio para a ciência forense.

\section{PARTE EXPERIMENTAL}

\section{Materiais e métodos}

Todos os reagentes e solventes que foram utilizados não receberam purificação adicional. Foram utilizados no processo de síntese, o ácido tereftálico (1,4- $\left.\mathrm{H}_{2} \mathrm{BDC}\right)$, óxido de gadolínio $\left(\mathrm{Gd}_{2} \mathrm{O}_{3}\right)$ e óxido de európio $\left(\mathrm{Eu}_{2} \mathrm{O}_{3}\right)$ que foram obtidos da Sigma-aldrich. Já a $\mathrm{N}, \mathrm{N}$-dimetil formamida, ácido nítrico $\left(\mathrm{HNO}_{3}, 65 \%\right)$ e álcool etílico $(99,8 \%)$ foram obtidos da Vetec. 


\section{Síntese da solução precursora da MOF mista luminescente}

A síntese dos microscristais em solução precursora foi realizada a partir de uma rota de produção otimizada, com relação ao que foi abordado na proposta de síntese desenvolvida por Nascimento et al. ${ }^{18,19}$ As relações estequiométricas foram mantidas em consonância com o que foi proposto previamente proposto pelo mesmo autor, ${ }^{18,19}$ para podermos realizar uma análise comparativa. A abordagem de síntese otimizada apresentada utilizou $0,19 \mathrm{mmol}$ de óxido de gadolínio e 0,01 mmol de óxido de Európio (dopagem de 5\%), que foram adicionados em um béquer, e adicionaram-se, em seguida, $5 \mathrm{~mL}$ de $\mathrm{HNO}_{3}$ concentrado $98 \%$. O sistema foi mantido sob agitação constante até a solubilização dos referidos óxidos e a formação dos nitratos em situ. Após o sistema resfriar até a temperatura ambiente, foram adicionados 1,6 mmol de ácido tereftálico (ligante) e 7,5 mL de DMF (dimetilformamida). O ácido tereftálico é um ligante de baixo custo, que apresenta boa sensibilização dos íons $\mathrm{Eu}^{3+}$ na presença dos íons $\mathrm{Gd}^{3+}$, pois promove o chamado efeito do átomo pesado, que promove o aumento do acoplamento spin-órbita à medida que o número átômico aumenta. ${ }^{18} \mathrm{O}$ sistema foi mantido sob agitação constante à temperatura ambiente até homogeneização da mistura. Foram adicionados, posteriormente, $2,5 \mathrm{~mL}$ de etanol, gota a gota, em alíquotas de $0,5 \mathrm{~mL}$ para atuar como agente precipitante, em intervalos de 1 minuto para induzir a nucleação de cristais. Por fim, o sistema foi mantido sob agitação constante à temperatura ambiente durante 20 minutos e a solução resultante foi reservada para atuar como tinta fotoluminescente.

\section{Obtenção dos cristais da MOF mista luminescente}

Uma alíquota de $5 \mathrm{~mL}$ da solução precursora foi centrifugada a $3600 \mathrm{rpm}$ durante 5 minutos. Os cristais obtidos foram filtrados e ficaram em um dessecador por 48 horas, para realizarmos comparações dos espectros de emissão obtidos, antes e após serem depositados nos substratos de papel.

\section{Padronização de substratos de papel com caneta}

A solução precursora foi inserida na caneta de tinta líquida pilot board master com ponta média e a tinta foi depositada nos substratos de papel. As tintas submetidas nos substratos foram deixadas secar por 5 minutos, tempo necessário para ocorrer o processo de secagem nos substratos de papel e ocorrer a deposição dos cristais da SP_Gd/EuMOF.

\section{Caracterização das amostras}

O espectro de excitação e emissão, foram obtidos a 298 K num espectrofluorímetro Fluorolog Horiba Jobin Yvon com fontes de excitação de lâmpadas de xenônio $(450 \mathrm{~W})$ contínua e pulsada. Após a deposição da SP_Gd/EuMOF e posterior secagem, promoveuse excitação através de um aparelho de ultravioleta portátil no $\lambda=254 \mathrm{~nm}$, para obtenção do material luminescendo nos substratos.

\section{RESULTADOS E DISCUSSÃO}

Na Figura 1, é apresentado o espectro de excitação dos cristais da SP_Gd/Eu-MOF $\left(\lambda_{\text {emissão }}=614 \mathrm{~nm}\right)$. O espectro exibe picos estreitos entre 350 e $550 \mathrm{~nm}$, atribuídos as transições f-f caracteristicas do íons európio. Como as transições intraconficuracionais $f-f$ dos íons $\mathrm{Gd}^{3+}$ estão acima da maioria dos níveis tripletos dos ligantes orgânicos, as emissões características desses centros metálicos não podem ser observadas. ${ }^{18}$ Além disso, observa-se uma banda larga entre 252 e
$350 \mathrm{~nm}\left(\lambda_{\text {máximo }}=300\right.$ e $\left.323 \mathrm{~nm}\right)$, atribuídas a estados excitados do ligante, majoritariamente, ao processo eletrônico $S_{1} \leftarrow S_{0}\left(\pi^{*} \leftarrow \pi\right)$ no ligante orgânico (1,4-BDC). Essas duas bandas são ligeiramente deslocadas em comparação com as bandas do ácido tereftálico puro (1,4-H $\left.\mathrm{H}_{2} \mathrm{BDC}\right)$, como consequiência da coordenação do centro metálico através do ligante. ${ }^{19} \mathrm{~A}$ grande diferença de intensidade da banda do ligante, com relação as transições intraconfiguracionais $f-f$, revela que o mesmo atua como um sensibilizador eficiente da luminescência.

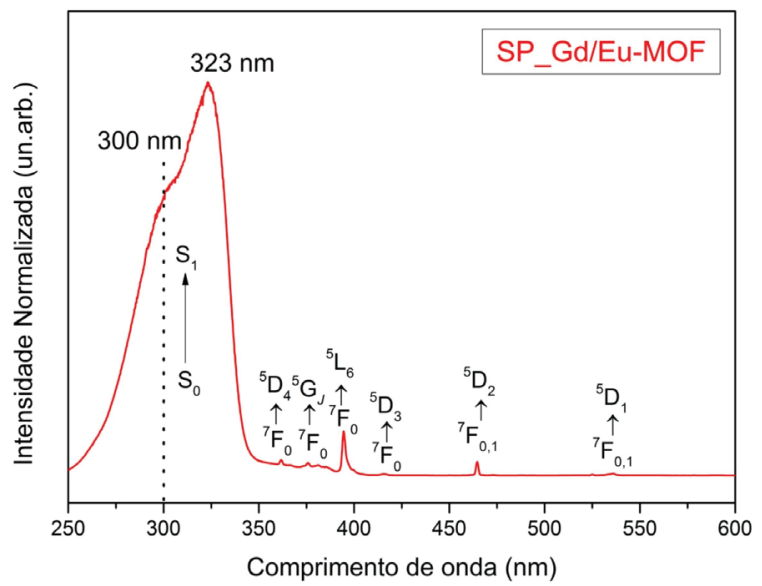

Figura 1. (a) Espectro de excitação do cristal SP_Gd/EuMOF $\left(\lambda_{\text {emissão }}=614 \mathrm{~nm}\right)$, obtido a temperatura ambiente

Na Figura 2 são apresentados os espectros de emissão característicos de cristais de SP_Gd/EuMOF (linha sólida preta) e o espectro de emissão desse mesmo material após ter sido depositado na folha de papel (linha sólida vermelha), ambos
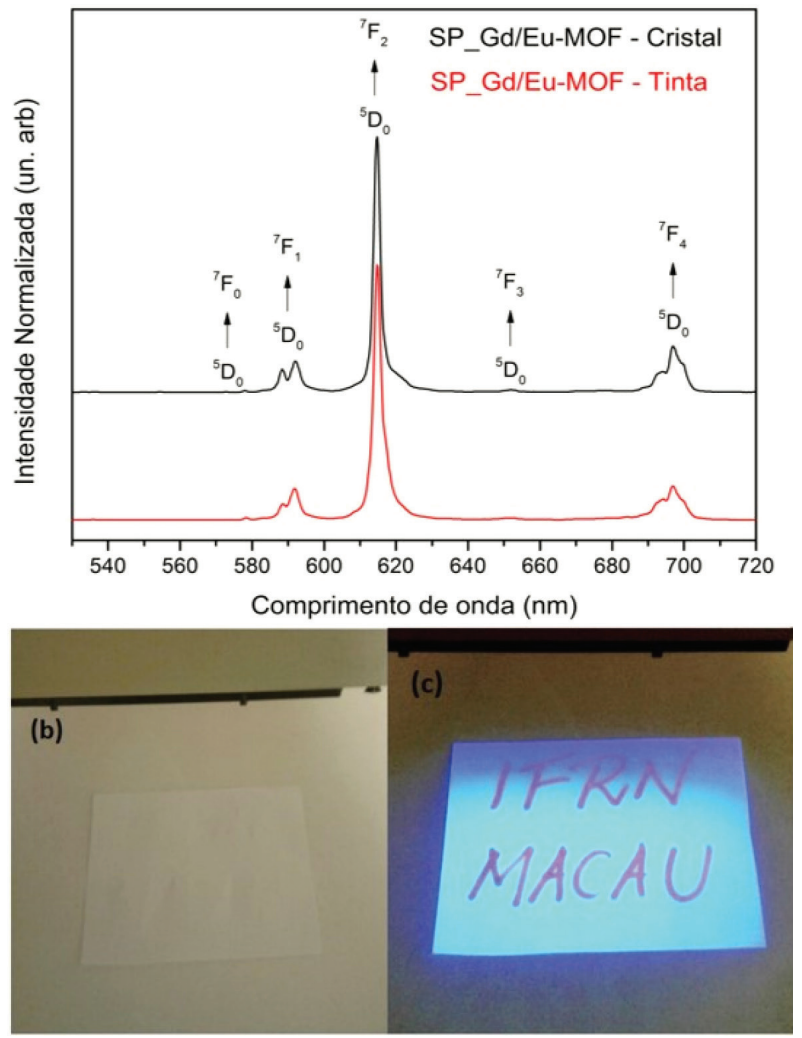

Figura 2. (a) Espectros de emissão do cristal SP_Gd/EuMOF (linha sólida preta) e Tinta fotoluminescente SP_Gd/Eu-MOF (linha sólida vermelha). Folhas com tinta fotoluminescente SP_Gd/EuMOF depositado através de caneta de tinta líquida exposta (b) a luz visível (c) a radição $U V(\lambda=254 \mathrm{~nm})$ 
obtidos à temperatura ambiente sob $\lambda=254 \mathrm{~nm}$ (a). Além disso, são apresentadas fotografias de folhas de papel riscadas com a tinta fotoluminescente SP_Gd/Eu-MOF, exposta a luz visível (b) e radiação $\mathrm{UV}(\lambda=254 \mathrm{~nm})(\mathrm{c})$.

O espectro de emissão do cristal da SP_Gd/Eu-MOF e das regiões riscadas com a tinta $\mathrm{SP} \_\mathrm{Gd} / \mathrm{Eu}-\mathrm{MOF}$ nas folhas, exibem bandas finas características das transições no íon $\mathrm{Eu}^{3+},{ }^{5} \mathrm{D}_{0} \rightarrow{ }^{7} \mathrm{~F}_{\mathrm{j}}$ (Figura 2a). ${ }^{20}$ Observa-se, em ambos espectros, a presença da transição ${ }^{5} \mathrm{D}_{0} \rightarrow{ }^{7} \mathrm{~F}_{0}$ em $576 \mathrm{~nm}$, indica que o íon $\mathrm{Eu}^{3+}$ está inserido em um um ambiente de baixa simetria sem centro de inversão do tipo $\mathrm{C}_{\mathrm{nv}}$, $\mathrm{C}_{\mathrm{n}}$ ou $\mathrm{C}_{\mathrm{s}} \cdot{ }^{21}$ Além disso, a transição ${ }^{5} \mathrm{D}_{0} \rightarrow{ }^{7} \mathrm{~F}_{2}$ de dipolo elétrico, a qual é hipersensível a mudanças na primeira esfera de coordenação, tem intensidade de transição muito maior que a transição ${ }^{5} \mathrm{D}_{0} \rightarrow{ }^{7} \mathrm{~F}_{1}$, que é uma transição de dipolo magnético. Esse comportamento corrobora com o que foi explicitado, sobre a ausência de simetria sem centro de inversão dos íons $\mathrm{Eu}^{3+} \cdot{ }^{22,23}$ Os espectros apresentam perfis muito parecidos, indicando que os cristais depositados sobre a superfície do papel são idênticos aos cristais de SP_Gd/Eu-MOF. As Figuras 2b-2c demonstram que a técnica de deposição das "tintas" fotoluminescentes (SP_Gd/Eu-MOF) em papel por canetas de tinta líquida de forma manual permite a criação de imagens ocultas úteis invisíveis a olho nu, capaz de atuar como etiqueta de segurança.

Além das folhas de papel comum, foi realizada a deposição em folhas de papel timbrado do Instituto Federal de Educação, Ciência e Tecnologia do Rio Grande do Norte (IFRN) (Figura 3), para simular a codificação de um documento oficial da instituição (Figuras 3a-3b), sendo que nele foi realizado um teste de abrasividade com uma borracha sob a tinta fotoluminescente (Figuras 3c-3d).

Nas folhas de papel timbrado, a tinta SP_Gd/Eu-MOF adicionada na parte inferior do documento (Figuras 3a-3b) também se mostrou eficaz, sendo capaz de codificar esses documentos, quanto a sua origem e integridade. Foi realizada uma deposição no corpo do mesmo documento com a sigla da instituição e as iniciais do campus

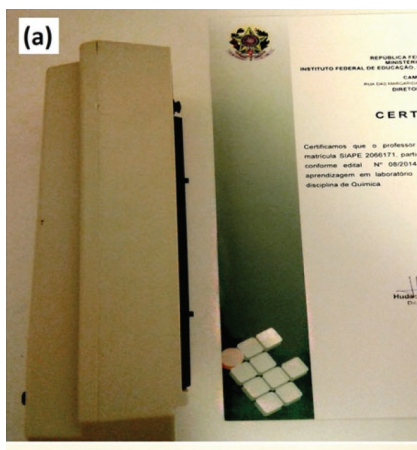

(c)

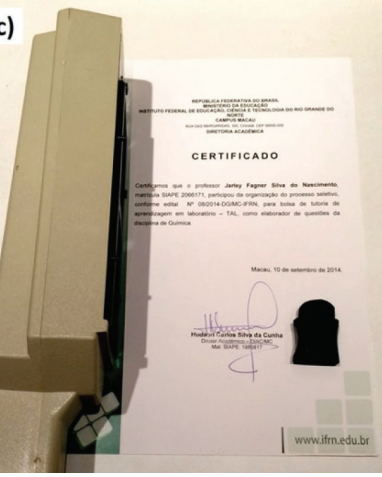

(b)

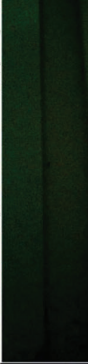

(d)

Figura 3. Folhas de papel timbrado com tinta fotoluminescente SP_Gd/ Eu-OF depositado através de caneta de tinta líquida exposta (a) a luz visivel (b) a radição $U V(\lambda=254 \mathrm{~nm})$ (c) teste de abrasividade com borracha sob luz visivel $(d)$ sob radição $U V(\lambda=254 \mathrm{~nm})$
(IFRN/MC - Instituto Federal de Educação, Ciência e Tecnologia do Rio Grande do Norte/Macau) (Figuras 3c-3d). Após a secagem, foi realizado um teste de abrasividade friccionando uma borracha no local, e após excitarmos o local onde foi depositada a tinta fotoluminescente, a mesma permaneceu aderida, mostrando interação satisfatória com o substrato.

\section{CONCLUSÕES}

Nesse estudo, foi apresentada uma estratégia eficiente para a deposição de tintas fotoluminescentes em substratos de papel de baixo custo, para codificação de documentos oficiais, utilizando canetas de tinta líquida de fácil obtenção no mercado. A tinta fotoluminescente SP_Gd/Eu-MOF apresentou intensidade luminescente na cor vermelha satisfatória, quando submetida a radiação $U V(\lambda=254 \mathrm{~nm})$, facilmente observada a olho nu. A tinta SP_Gd/Eu-MOF, mesmo após o processo abrasivo, exibiu boa adesão e estabilidade, confirmando seu potencial para ser utilizada como prova de autencidade. Além disso, sua metodologia de deposição se mostrou simples e rápida e não promoveu qualquer dano aos substratos de papel, sendo uma alternativa à deposição de MOFs luminescentes via impressoras a jato de tinta.

\section{AGRADECIMENTOS}

Os autores agradecem ao Instituto Federal de Educação, Ciência e Tecnologia do Rio Grande do Norte (IFRN), à pro-reitoria de pesquisa e inovação desse mesmo instituto (PROPI/IFRN) por financiar esta pesquisa, e à Universidade Federal do Rio Grande do Norte (UFRN), em especial ao Laboratório de Materiais Multifuncionais e Experimentação Numérica (LAMMEN).

\section{REFERÊNCIAS}

1. Hoffmann, F.; Fröba, M.; Chem. Soc. Rev. 2011, 40, 608.

2. Ariga, K.; Ji, Q.; Hill, J. P.; Bando, Y.; Aono, M.; NPG Asia Mater. 2012, 4, 17.

3. da Luz, L. L.; Milani, R.; Felix, J. F.; Ribeiro, I. R.; Talhavini, M.; Neto, B. A.; Júnior, S. A.; ACS Appl. Mater. interfaces 2015, 7, 27115.

4. Zhou, J.; Li, H.; Zhang, H.; Li, H.; Shi, W.; Cheng, P.; Adv. Mater. 2015, 27, 7072 .

5. Gangu, K. K.; Maddila, S.; Mukkamala, S. B.; Jonnalagadda, S. B.; Inorg. Chim. Acta 2016, 446, 61.

6. Xu, H.; Hu, H. C.; Cao, C. S.; Zhao, B.; Inorg. chem. 2015, 54, 4585.

7. Qin, J. S.; Bao, S. J.; Li, P.; Xie, W.; Du, D. Y.; Zhao, L.; Su, Z. M.; Chem. -Asian J. 2014, 9, 749.

8. da Silva, G. G.; Silva, C. S.; Ribeiro, R. T.; Ronconi, C. M.; Barros, B. S.; Neves, J. L.; Júnior, S. A.; Synth. Met. 2016, 220, 369.

9. Barros, B. S.; De Oliveira, R. S.; Kulesza, J.; Melo, V. R. M.; Melo, D. M. A.; Alves Jr, S.; J. Phys. Chem. Solids 2015, 78, 90.

10. Furukawa, H.; Cordova, K. E.; O’Keeffe, M.; Yaghi, O. M.; Science 2013, 341, 6149.

11. Zhang, C.; Wang, B.; Li, W.; Huang, S.; Kong, L.; Li, Z.; Li, L.; Nat. Commun. 2017, 8, 1138.

12. Ma, Y.; Wang, S.; Wang, L.; TrAC -- Trends Anal. Chem. 2015, 65, 13.

13. Ren, X. Y.; Lu, L. H.; Chin. Chem. Lett. 2015, 26, 1439.

14. Zhuang, J. L.; Ar, D.; Yu, X. J.; Liu, J. X.; Terfort, A.; Adv. Mater. 2013, $25,4631$.

15. D'álmeida, M. L. O.; Koga, M.; Granja, S.; Documentoscopia: o papel como suporte de documentos, $1^{\mathrm{a}}$ ed., IPT: São Paulo, 2015.

16. Jiang, K.; Zhang, L.; Lu, J.; Xu, C.; Cai, C.; Lin, H.; Angew. Chem., Int. Ed. 2016, 55, 7231 .

17. Wang, Y. M.; Tian, X. T.; Zhang, H.; Yang, Z. R.; Yin, X. B.; ACS Appl. Mater. Interfaces 2018, 10, 22445. 
18. Nascimento, J. F. S.; Barros, B. S.; Kulesza, J.; Oliveira, J. B. L.; Leite, A. K. P.; Oliveira, R. S.; Mater. Chem. Phys. 2017, 190, 166.

19. Nascimento, J. F. S.; Araújo, A. M. U.; Kulesza, J.; Farias Monteiro, A. F.; Júnior, S. A.; Barros, B. S.; New J. Chem. 2018, 42, 5514.

20. Binnemans, K.; Chem. Rev. 2009, 109, 4283.
21. Chen, B.; Wang, L.; Xiao, Y.; Fronczek, F. R.; Xue, M.; Cui, Y.; Qian, G.; Angew. Chem., Int. Ed. 2009, 48, 500.

22. De Oliveira, R. S.; De Brito, B. S.; Kulesza, J.; Alves-Jr, S.; Barros, B. S.; Ceram. Int. 2017, 43, 8276.

23. Zhu, T.; Chen, P.; Li, H.; Sun, W.; Gao, T.; Yan, P.; Phys. Chem. Chem. Phys. 2015, 17, 16136. 